\title{
Location-Based Adaptive Mobile Learning Research Framework and Topics
}

\author{
Qing Tan ${ }^{1}$, Kinshuk ${ }^{1}$, Yen-Hung Kuo ${ }^{2}$, Yu-Lin Jeng ${ }^{2}$, \\ Po-Han $\mathrm{Wu}^{2}$, Yueh-Min Huang ${ }^{2}$, Tzu-Chien Liu ${ }^{3}$, and Maiga Chang ${ }^{1}$ \\ Athabasca University, Canada ${ }^{1}$ \\ National Cheng Kung University, Taiwan (R.O.C.) ${ }^{2}$ \\ National Central University, Taiwan (R.O.C.) ${ }^{3}$ \\ qingt@athabascau.ca
}

\begin{abstract}
Location-based adaptive mobile learning utilizes a unique feature: location awareness of mobile devices in regards to other online learning to implement location-based functionalities or features to create adaptive and collaborative learning in mobile learning environment. There are many important research issues and technical challenges in the location-based adaptive mobile learning systems, including the assessment of learning in the mobile environment. In this paper, we present the research framework and topics related to the undergoing research project at Athabasca University towards the development of robust and effective location-based adaptive mobile learning systems. In addition, we also brief some the major research outcomes achieved so far within the project's research framework.
\end{abstract}

\section{Introduction}

With the matured infrastructure and the wide coverage of cellular communication networking and the rapid development of advanced mobile technologies, mobile phones have been used far beyond their telecommunication capabilities and more as powerful data communication devices. The number of mobile devices accessing the Internet has been increasing rapidly over recent years. Studies have shown that in the United States the numbers have grown significantly, demonstrating a quarterly growth increase of $154 \%$ in Q4 of 2007 versus the same period in 2006 [1]. Similarly, another study of mobile Internet usage identified that in Japan, there were over 53 million users that accessed the Internet on their mobile devices in June of 2007 [2].
Mobile computing has become a very important sector in computing science. Subsequently, mobile learning has been adopted by many learners and it has been considered as an effective way for learning anytime and anywhere. Location-based services (LBS) are one of the fundamental components in the cellular communication network. Furthermore, mobile phones with built-in GPS receiver or other type of A-GPS services are widely available in the market. Utilizing the mobile phones' location awareness into mobile applications has become a new trend, which is greatly changing our daily lives. However, there are many critical issues and technical challenges related to the location-based adaptive mobile learning. In particular, the mobile learning applications require mobile software to run on mobile phones in order to get mobile learner's updated location data and to interact with mobile phone's native functionalities and hardware. Among the natural constraints of a mobile phone, such as scarce resource limitations, heterogeneity and use of multiplatform mobile operating systems, user/device interaction limitations, high cost of data communications, and device mobility and portability, the primary challenge is to ensure that the mobile software can work across platforms [3][4][5]. From mobile learning prospective, it is very important to utilize the mobile phone's characteristics, features and functionalities to develop the right applications in order to facilitate the learning. Furthermore, from pedagogy view point, applying the technologies to learning is to enhance the education. To properly evaluate the location-based adaptive mobile learning and its systems is a way to generally improve the systems and finally achieve the overall research goal. Therefore, properly identifying the critical issues, finding right research topics, and building the right 
research framework will be greatly beneficial to the overall research and development as well as to the mobile learning evaluation from technology and pedagogy prospective.

In this paper, we also present some of the major research outcomes achieved so far within the project's research framework. The first outcome presentation is to show how to use mobile phone hardware: camera and GPS receiver to create location based learning contents. For example, with the learner agent - mobile client software, a mobile learner can take a picture attached with its location information to create an authentic example for learning. The location information indicates where the authentic example was taken [6]. The mobile user can annotate and share the example. Other students can learn from the example or go to the location to experience the example. The second outcome presentation is to demonstrate how to employ the location awareness of a mobile phone to serve for mobile learners in mobile learning environment. A location-based dynamic grouping algorithm creates learning groups by identifying the individual mobile learners' geographic locations and other learning factors [7]. This algorithm will eventually generate online Mobile Virtual Campus where mobile learners are grouped based on their geographic location, learning profiles and learning styles, or learning interests in the mobile learning environment. The third outcome presentation is to address the challenges of mobile computing and to present the design principles. With respect to the nature of mobile phones, the proposed five design principles could be applied to any client mobile software required by a mobile application system [8]. The fourth outcome presentation is to provide a solution to deal with the challenges of mobile computing. A mobile computing architecture for multi-platform adaptation consists of an installation agent and two mobile computing solutions to accommodate different mobile platforms [9]. The proposed architecture provides a practical and effective solution for multiplatform adaptation in compliant with the design principles in mobile computing.

In the next section, the research framework and topics on the location-based technologies and mobile computing for adaptive mobile learning are presented. In the following four sections, the research outcomes are discussed within the research framework. The last section concludes this paper.

\section{Research Framework and Topics}

To successfully achieve the research goals of location-based adaptive mobile learning, our research framework consists of three layers shown in Fig. 1: the technology foundation, the locationbased mobile learning application, and the study of mobile learning and technology impact. The technology foundation research will focus on location-based technologies and mobile computing. The location-based mobile learning application research aims to apply location awareness of mobile devices and other location technologies to develop algorithms and applications in mobile learning environment in order to facilitate the technologies to apply for mobile learning, such as dynamic grouping and mobile virtual campus, mobile learner social networks, and location based learning content. Mobile learning will be enhanced with the effective and adaptive mobile learning infrastructures and with the newest technologies. The top layer of this research framework is to study and evaluate the pedagogical effectiveness of the location-based adaptive mobile learning; to understand the technologies' impact on mobile learning, to analyze mobile learners' behaviors and patterns in mobile learning environment; to identify the strengths and weaknesses of mobile learning; to provide possible mobile learning solutions and suggestions; and to explore trends associated with the future of mobile learning.

There are various research topics within this research framework. They are categorized and briefly described as follows.

\section{I: The Technology Foundation}

- GPS Location Technologies in Mobile Devices

To study A-GPS technology and its availability in mobile environment and to identify the right approaches of retrieving GPS data and the possible applications

- Cellular Network Positioning Issues: Approaches, Limitations, and Applications To investigate the different cellular position methodologies and to find their straights and 


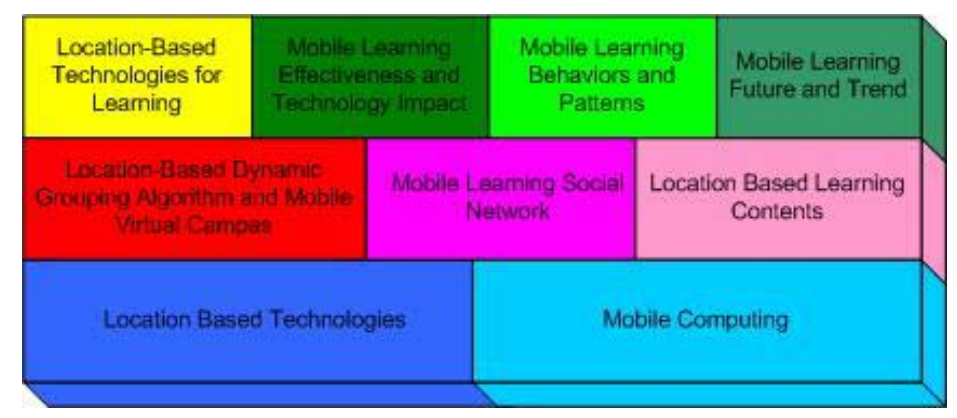

Figure 1: Location-Based Adaptive Mobile Learning Research Framework

weakness and how to integrate the technology into mobile learning applications

- Q.R Code and RFID for Location Based Applications: Scenario and Infrastructure To understand Q. R. Code and RFID as location components and to evaluate the effectiveness of using Q. R. Code and RFID in location-based applications and to explore application scenarios for location-based mobile learning infrastructure

- $\quad$ Client Mobile Software Design Principles

To study the mobile computing limitations and to provide five design principles in client mobile application development in order to guide the mobile application development and to provide mobile software evaluation criteria

- $\quad$ Mobile Computing Architecture for Multiplatform Adaptation

To study mobile computing architecture in order to provide an effective and practical solution for multiplatform adaptation issues in mobile computing

- Mobile Devices Application Running Environments: Current and Trend

To study a variety of mobile devices and mobile computing platforms and to understand their running environments and to find the possible evolution of mobile computing and mobile devices

- Mobile Computing Issues: Human-Machine Interaction and Security

To study possible human-machine interactions in order to develop high performance and easily usable mobile applications and to identify the security issues and solutions in mobile computing and applications
- Intelligent Learning Algorithm for Enhancement of Location Based Mobile Learning System

To study intelligent learning algorithms in order to develop an intelligent learning mechanism in order to continuous upgrade and calibrate the location data and to improve the location accuracy in location-based mobile application systems

\section{II: The Location-Based Mobile Learning Applications}

- Location-Based Dynamic Grouping Algorithm in mLearning Environment To develop a location-based dynamic grouping algorithm by integrating individual mobile learner's geographic location and learning profile and learning style or learning interests

- Mobile Virtual Campus for Location-Based Adaptive mLearning System

To develop a location-based adaptive mobile learning system with mobile virtual campus for online mobile learners by using the location-based dynamic group algorithm

- Location-Based Dynamic Grouping Algorithm mLearning System Evaluation To study the developed and implemented location-based adaptive mobile learning system and to analyze and evaluate the performance, effectiveness, and usability of the system

- Social Closeness in Location-Based Adaptive mLearning System

To propose a concept of social closeness and to identify the social closeness among the mobile learners and apply the social closeness 
concept in the location-based adaptive mobile learning system

- Mobile Social Network for Mobile Learners To study mobile social network and to identify mobile social network's characteristics, advantages, limitations, and issues in regard to other online social networking

- $\quad$ Fuzzy Logic and Measurement for Computing Non-quantified Data in Mobile Learning To use fuzzy logic for the non-quantified data in mobile learning environment and to provide proper measurements and calculations for mobile computing and evaluation

- Creation and Delivery of Location-Based Learning Contents in mLearning Systems To study how to create and delivery locationbased learning contents and to find and provide optimal attributes of location-based learning objects and to study the significance of location-based learning contents in mobile learning environment

\section{III: Study Mobile Learning and Technology Impact}

- $\quad$ Location-Based Technologies in Learning To study the rationale behind location-based technologies for learning and the effectiveness and application areas of location-based technologies for learning

- Effectiveness of Mobile Learning: Strength and Weakness

To study the effectiveness of mobile learning and to identify the strength and weakness of mobile learning from technology and pedagogy prospective and to provide proper suggestions and advices for mobile learner and mobile learning system development

- Mobile Learners' Behaviors and Patterns in Mobile Learning

To study mobile learners' behaviors and to find the learning patterns in order to build mobile learning models and to develop effective mobile learning system

- Grouping and Social Closeness in Mobile Learning Environment

To evaluate the group learning and social closeness in mobile learning environment in order to identify how the collaborative learning is effective in mobile learning and to find the use of the grouping algorithm and social closeness in mobile environment

- Mobile Learning: Current, Trend, and Future To study the driving power of the evolution of mobile learning and to find the technological impact on mobile learning and to predicate the future of mobile learning and to identify the position of the mobile learning in modern education

\section{Collaborative Creation of Authentic Examples with Location Data}

According to Figure 2, there are three agents that work and interact in the learning environment: a Learner Agent, a Location-aware Agent, and a Resource Agent. In this study, we will especially focus on the Learner Agent, which is installed into students' cellular phones and is responsible for reporting students' location information to the Location-aware Agent, as well as cooperating with the Resource Agent to manage the authentic examples.

In this work, we have developed the Learner Agent in J2ME programming language, and the agent program can run on any GPS-enabled phone with a Java Virtual Machine (JVM). Hereinafter, we are going to introduce the Learner Agent in related to agents' characteristics.

- Proactive: The Learner Agent's goal can be divided into three sub-tasks: 1) Monitoring the change of the folder; 2) Attaching the location information and time stamp to a new incoming authentic example, and then uploading it to the server; 3) Periodically reporting student's location to the Location-aware Agent. The detail task of the Learner Agent is shown in Figure 2.

- Personalized: The three sub-tasks of Learner Agent can be turned on and off individually. Moreover, the student can specify any folder in her cellular phone.

- Persistent: Generally speaking, the Learner Agent will run continuously until it to be turned off.

- Reactive: The Learner Agent can automatically adjust the GPS receiving interval and the location reporting interval to prevent power drop. 


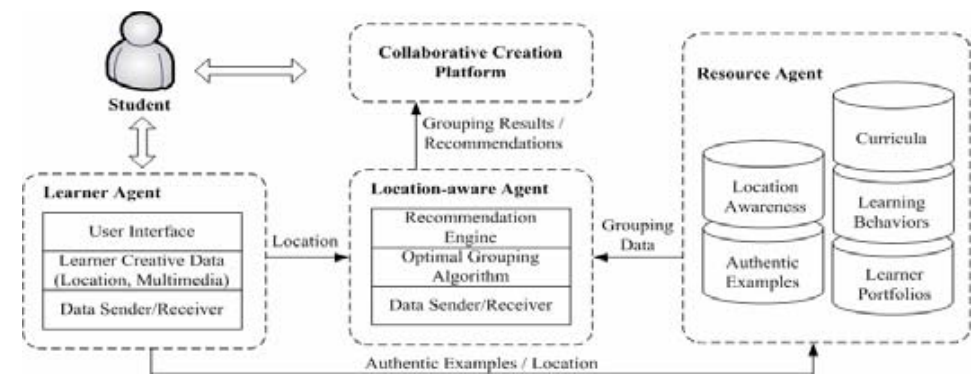

Figure 2: Multi-agent system architecture

- Social Behavior: The Learner Agent will interact with the Location-aware Agent and the Resource Agent while exchanging data.

- Autonomous: The Learner Agent will carry on its duty automatically and continuously in the background. The entire process does not need a student to involve except for initial installation.

- Trustworthy: A student can always anticipate and believe that the Learner Agent will only do the things that $s /$ he wants it to do. Except for the Learner Agent, the other major contribution of this work is the implementation of the mobile webbased collaborative creation platform. On the platform, students can annotate and publish their own authentic examples, and other peer can browse these examples.

\section{Location-Based Dynamic Grouping Algorithm in mLearning Environment}

When a learner initiates the grouping algorithm it will automatically generate a virtual proximity circle with a default radius for that learner. The center of the circle is the learner and, initially, s/he is designated a group leader. There are three possible grouping outcomes for the particular learner at a particular time and location. 1. Other learners, known to the system and who are within the virtual proximity circle, could be asked to join the imitating learner's group. 2. The learner could be assigned to an existing group. 3. The learner could simply be designated as being the sole member of his/her group.

Within the proposed location based grouping algorithm, there are many rules that have been predefined in order to create proper groups and to optimize the grouping process and performance.

The proposed location based grouping algorithm enables learners to be grouped together based on

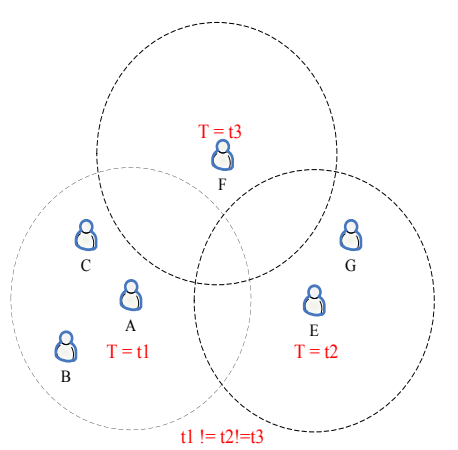

Figure 3: Several possible grouping statuses

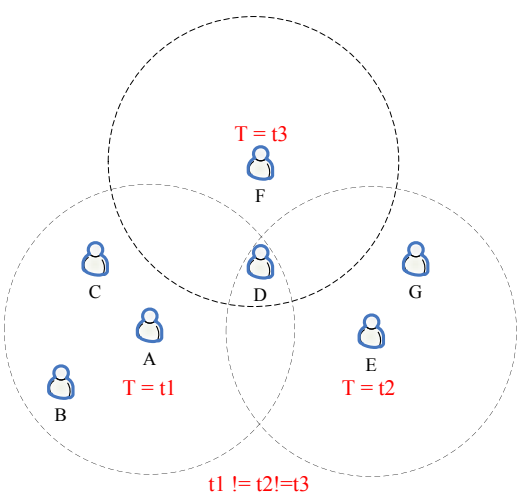

Figure 4: Several possible situations

their location information. However, for learning in a mobile environment, the purpose of the grouping is to enhance learning using all known factors. Simply using the location information may not result in an optimal solution from the learner's perspective. To enhance the algorithm and create true Mobile Virtual Campuses (MVCs) learning factors are taken into account. These learning factors can be characterized by the learners' 
learning profile; the learner's learning style and their learning interests.

Firstly, a learner's background, their age, certain aspects of their learning history and previous learning performance and educational level could have influence on the learning behavior. For our work we consider the learner's age, the subject that is to be learnt and a measure of the learner's learning progress as the key elements of the learner's profile.

Secondly, we consider learning style as an important feature. Learners with a similar learning style tend to have more interactions with one another during their learning experience. Learners grouped together and who have a similar learning profile and style tend to profit more from the interaction in situations where collaborative learning is necessary. The proposed location based grouping algorithm used the Felder-Silverman learning styles model [10] to analyze learners' learning styles. Furthermore, Felder and Soloman proposed an Index of Learning Styles (ILS) Questionnaire to assess users' learning styles. The proposed grouping algorithm obtains a learner's learning style value by asking them to fill out the ILS questionnaire. The ILS methodology results in a four dimensional 4-tuple with element values ranging from -11 to 11 .

Finally, we consider the learner's interest as a key characteristic within the context of a learning group. Interest is defined as a content-specific motivational characteristic encompassing the depth of text comprehension, the use of learning strategies, and the quality of the emotional experience while learning [11]. The premise is that learners with similar interests, in a group, can facilitate their learning when pursuing specific learning objectives. Our proposed algorithm captures learners' interests simply by asking them to pick or type in their interests along the dimensions mentioned above. A learner can have more than one interest.

Let $\omega$ be the weighting value of each feature. Accordingly, $\omega_{l}$ is the weighting value of location based grouping algorithm, $\omega_{p}$ is the weighting value of learner profile, $\omega_{s}$ is the weighting value of learner learning style, and $\omega_{i}$ is the weighting value of learner interests. The weighting value summation of each process should be 1 . Thus, $\omega_{l}+\omega_{p}+\omega_{s}=1$ or $\omega_{l}+\omega_{i}=1$. To emphasize the importance of location information and learner's interests, we set $\omega_{l}=\omega_{i}=0.5$.

Let $S$ be the final score of grouping result. The score function of $\mathrm{S}$ can be represented as the following two equations:

$$
\begin{aligned}
& \mathbf{S}\left(\mathbf{L}_{n}, \mathbf{C}_{m}\right)=\omega_{1} \mathbf{S L}_{l}\left(\mathbf{L}_{n}, \mathbf{C}_{m}\right)+\omega_{p} \mathbf{S L}_{p}\left(\mathbf{L}_{n}, \mathbf{C}_{m}\right)+\omega_{s} \mathbf{S L}_{s}\left(\mathbf{L}_{n}, \mathbf{C}_{m}\right) \\
& \mathbf{S}\left(\mathbf{L}_{n}, \mathbf{C}_{m}\right)=\omega_{1} \mathbf{S L}_{l}\left(\mathbf{L}_{n}, \mathbf{C}_{m}\right)+\omega_{p} S_{L_{p}}\left(\mathbf{L}_{n}, \mathbf{C}_{m}\right)
\end{aligned}
$$

In above equations, the $S L_{l}$ value is an important factor in calculating the final score, $S$. This emphasizes the importance of the location factor in the grouping algorithm. The value of the location similarity level $S L_{l}$ will be assigned to 1 when the learner $n$ is within range of the MVC $m$. However, if the final score, $S$, implies more than one match with existing MVCs we further compare the $S L_{l}$ values (between 0 to 1 ) to provide a best recommendation for grouping. If the $S L_{l}$ values are the same, the MVCs will be presented to the learner for her/his decision as to which group to join.

\section{Client Mobile Software Design Principles for mLearning Systems}

In the paper [8], there is the detailed discussion about the client mobile software design challenges as well as the rationale of the following design principles.

Multiplatform Adaptation: Client mobile software must work for multi-platforms. For example, the location-based dynamic grouping algorithm provides grouping suggestions to the mobile learners. The algorithm requires client-side software to run on the learners' mobile phones. The client application gets the learners' geographic location data. As a result, the mobile learners will download and run the client mobile software on their own MIDP 2.0 ready phones. The client mobile application has to work for a variety of phones on multi-platforms.

Little Resource Usage: The resource constraints of mobile phones mean that any application should employ the built-in functions and features of the phone. The application must be designed to use the computation capabilities of the phone as little as possible. The phone is thus adopting the role of a mobile terminal but not a mobile workstation. In adaptive mobile learning system, for example, uses 
client software, running in background mode to acquire, package, and send the phone's location data to the application server. The bulk of the computation takes place on the server side.

Little Human/Device Interaction: A mobile user needs to interact with his/her mobile phone to perform necessary actions. Because of the small screen or/and keypad, human/device interaction is inconvenient and even difficult in some cases. A design principle is, therefore, the less human interaction is required the better the mobile application is.

Small Data Communication Bandwidth Usage: In order to dedicate most of the computational load to the server side more communication between the client and the server may be required. Mobile data communication, via the cellular network, is very expense and consumes phone's scarce resources, such as battery power. A design principle for a client-server mobile application system is that there should be as little communication between the client phone and the server computer as possible.

No Additional Hardware: The mobility and portability of mobile phones is of great utility to users. Currently available phones have been integrated with much useful hardware and software to enhance the mobile phone's features. The mobile phone is not only a communications device but it also offers a powerful multifunctional tool for people's daily use. Any additional hardware that is required by a mobile application will reduce the compactness and portability of phone. It will also create extra cost for the user and give rise to the lack of compatibility issue. Consequently, the principle of no additional hardware is very important in the context of mobile applications.

\section{Mobile Computing Architecture for}

\section{Multi-platform Adaptation}

Based on our study and development experiences, we propose mobile computing architecture for multi-platform adaptation. This architecture consists of an installation agent and three adaptable mobile application computing solutions to accommodate different mobile platforms as shown in Figure 5. To implement the multi-platform adaptation architecture requires MIDP2 and CLDC1.1 ready mobile device.

With the multi-platform adaptation architecture, a mobile user need to go to a URL with the mobile web browser and to download the installation agent, a J2ME program that can run on any MIDP2 and CLDC1.1 ready mobile device. After installing and running the installation agent program, the mobile device's platform information will be automatically sent to the installation server. In the server, the mobile device's platform will be assessed with its platform information based on the

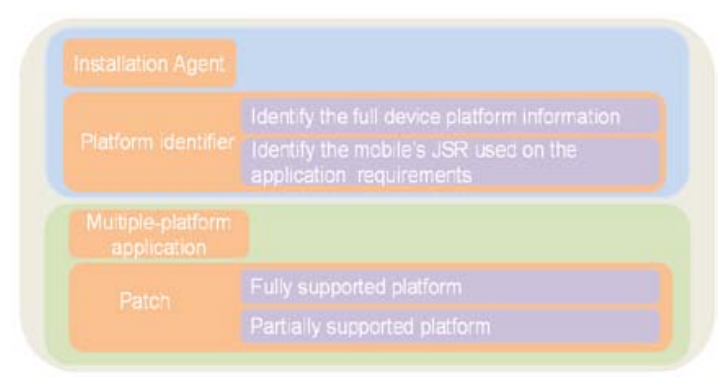

Figure 5: Multi-platform adaptation architecture

running environment requirements of the mobile application software. The assessment will identify the mobile device's platform as a fully supported platform or a partially supported platform. According to the assessment results, necessary patch programs and matching mobile application software will be provided for the particular mobile device's platform. Then the mobile user will be prompted to download the patch programs and mobile application software to achieve the multiplatform adaptation in mobile computing.

Mobile platform identification: In the multiplatform adaptation architecture, firstly, the installation agent is a J2ME program that can run on any MIDP2 and CLDC 1.1 ready mobile device without other Java APIs defined by optional Java Specification Requests (JSR). It detects a mobile device's operating system and other platform information and returns the detected information automatically to the installation server. Secondly, a platform identifier on the server will assess the information to identify the mobile device's platform and to find out the deficiency of the mobile device's Java Running Environment for running the mobile application software. The Installation agent workflow and its pseudo code are shown in Figure 6. 


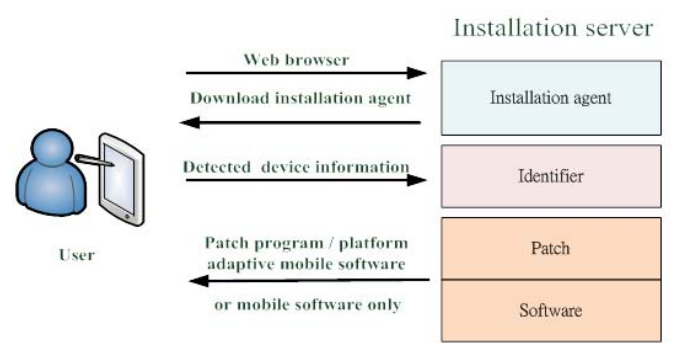

Figure 6: Installation agent workflow

Mobile platform adaptation: The platform identifier on the server assesses the identified mobile platform and compares with the running environment requirement of the mobile application software. Then, the platform identifier categorizes the mobile platform as fully supported platform or partially supported platform. A fully supported platform is fully compatible with the running environment required by the mobile application software. A partially supported platform is lack of some APIs defined by the JSRs required by the mobile application software in addition to MIDP2. According to identified platform, the platform identifier provides the necessary patch to the mobile platform and prepares adapted mobile application software to achieve the mobile platform adaptation.

To implement the multi-platform adaptation architecture, except to develop the architecture required installation agent and platform identifier, the solutions required development include one patch APIs for each mobile operating system to upgrade MIDP2 running environment and only one platform adapted mobile application software.

\section{Conclusion}

Location-based technologies can be applied to mobile learning applications. With the location awareness, mobile learning could be more adaptable and mobile learning system with the unique feature can greatly enhance the collaboration for online learners. Mobile computing is fundamental part of mobile learning application development. The Five mobile software design principles provide the guideline for the mobile software development as well as for the mobile application evaluation. There are a lot of researches that have to be done relative to mobile learning. Some of them could be very challenging. With the research, development, and use of mobile learning systems, mobile learning will play more and more important role in modern education.

\section{References}

[1] A. Lipsman. "Number of U.S. Computers Accessing the Internet via Mobile Broadband Soars 154 Percent in 2007" comScore From http://www.comscore.com/, March, 2008

[2] J. Gavin, "Mobile Phone Web Users Nearly Equal PC Based Internet Users in Japan." comScore. From http://www.comscore.com/, September, 2007

[3] C. Mascolo, L. Capra, and W. Emmerich, "Mobile computing middleware," In Advanced lectures on networking pp. 20-58, 2002

[4] D. Grigoras. "Challenges to the Design of Mobile Middleware Systems," In Parallel Computing in Electrical Engineering, International Symposium on pp. 14-19, 2006

[5] S. Vaughan-Nichols, "Wireless middleware: glue for the mobile infrastructure. Computer" 37(5), 18-20, 2004

6] Yen-Hung Kuo, Qing Tan, et al., "Collaborative Creation of Authentic Examples with Location for U-Learning" published on IADIS eLearning 2008

[7] Qing Tan, Yueh-Min Huang and Yu-Lin Jeng "Location-Based Dynamic Grouping Algorithm in Mobile Learning Environment”, 2009

[8] Qing Tan and Kinshuk "Client Mobile Software Design Principles for Mobile Learning Systems", International Journal of Interactive Mobile Technologies (iJIM), Vol3, No. 1, 2009

[9] Qing Tan, Po-Han Wu, Yu-Lin Jeng, Kinshuk, and Yueh-Min Huang "Mobile Computing Architecture for Multi-Platform Adaptation" IADIS Wireless Applications and Computing 2009

[10]R.M. Felder \& L.K. Silverman (1988). Learning and Teaching Styles in Engineering Education, from http://www.engr.ncsu.edu/, 2009

[11] Schiefele U. Interest learning and motivation. Educational Psychologist, 26, 299-323, 1991 